\title{
BMJ Open Bathing adaptations in the homes of older adults and their carers (BATH- OUT): a qualitative extended follow-up study with concurrent nested outcome assessments
}

\author{
Miriam Golding-Day (D , ${ }^{1}$ Phillip Whitehead ${ }^{2}$
}

To cite: Golding-Day M, Whitehead P. Bathing adaptations in the homes of older adults and their carers (BATH-OUT): a qualitative extended follow-up study with concurrent nested outcome assessments. BMJ Open 2020;10:e035701. doi:10.1136/ bmjopen-2019-035701

- Prepublication history and additional materials for this paper is available online. To view these files, please visit the journal online (http://dx.doi. org/10.1136/bmjopen-2019035701).

Received 14 November 2019 Revised 31 August 2020 Accepted 21 September 2020

Check for updates

(c) Author(s) (or their employer(s)) 2020. Re-use permitted under CC BY-NC. No commercial re-use. See rights and permissions. Published by BMJ.

${ }^{1}$ Division of Rehabilitation, Ageing \& Wellbeing, University of Nottingham, Nottingham, UK ${ }^{2}$ Department of Social Work, Education and Community Wellbeing, Northumbria University, Newcastle upon Tyne, UK

Correspondence to Miriam Golding-Day; miriam.golding-day@ nottingham.ac.uk

\section{ABSTRACT}

Objective We previously completed a nested qualitative interview study, as part of a feasibility randomised controlled trial with 21 older adults and five carers who had an accessible shower installed in their home. The objective of this study was to follow-up the participants approximately 24 months on.

Design This was an extended follow-up study comprising semi-structured interviews to explore the longer-term experiences of the older adults. To elaborate and add breadth to the findings these were supplemented with concurrent nested outcome assessment measures. Setting The study was conducted within one local authority City Council housing adaptations service. Participants Thirteen older adults (mean age: 76; SD: $6.87)$ and three carers from the original study completed the extended follow-up study.

Interventions The intervention in the original study was the provision of an accessible showering facility either by immediate provision or routine 4-month wait.

Results Findings were presented thematically with three themes identified: environment, autonomy with personal care and wider occupation. Improvements in the physical and social environment combined with greater autonomy in personal care were reported to impact more widely on older adults' occupations including other self-care activities and leisure. These are consistent with domains underpinning social care related quality of life particularly personal safety, cleanliness and occupation. The results of the outcome assessments support the qualitative themes demonstrating sustained improvements in quality of life, independence in daily living and reduced fear of falling. Conclusion This research suggests the positive lived experiences reported immediately after the installation of the accessible shower are still evident up to 28 months later in this cohort of older adults. Future research should investigate medium to longer-term outcomes with a more diverse sample.

Trial Registration ISRCTN14876332; Post-results.

\section{INTRODUCTION}

The home environment is an important factor affecting the mental health, well-being and
Strengths and limitations of this study

- This is the first study to explore the longer-term lived experiences of older adults who have had a bathing adaptation to install an accessible showering facility in their homes.

- The qualitative findings are supported by the results of the outcome measures which adds to the trustworthiness of the data and our analysis.

- The study was only conducted within a small nondiverse sample from one local authority.

- This study uses a developing embedded mixed methods approach which could enable researchers to model future studies.

quality of life for older adults. ${ }^{1}$ The onset of difficulties with everyday activities within the home is a seminal point in the life course for older adults indicative of possible functional decline and loss of independences. Housing adaptations are alterations to the fabric of the home that aim to remove the barriers that are causing difficulties in order to improve safe and/or independent functioning and prevent or delay the need for other services. Although housing adaptations have been identified as a 'top-ten' prevention intervention for older adults, ${ }^{2}$ there continue to be delays in the provision of adaptations across all housing tenures. ${ }^{3}$ A systematic review, ${ }^{4}$ found that there was good evidence for the effectiveness and cost effectiveness of minor adaptations (such as grab rails and altered thresholds) for preventing falls and particularly injuries caused by falls. ${ }^{5}$ Powell et al reported that major adaptations, such as ramps, stair lifts and accessible showers, have been less extensively studied. ${ }^{4}$ They concluded further studies were needed, particularly randomised controlled trials (RCTs) in UK settings and studies taking a longitudinal perspective. 
Furthermore, the role of the home environment in maintaining older adults' independence has received little attention in relation to service delivery. ${ }^{4}$

The onset of difficulties with the activity of bathing, defined as 'the inability to wash or dry one's whole body without personal assistance, ${ }^{6}$ has been shown to lead to an increased likelihood of disability with other activities of daily living for older adults, ${ }^{6}$ and for nursing home admissions. ${ }^{7}$ Bathing adaptations are bathing/bathroom specific "permanent alterations carried out to a building with the aim of making it more suitable for a disabled person' ${ }^{8}$ This usually involves the removal of the bath and replacement with an accessible 'level-access' shower. These are the most common type of major housing adaptation for older adults, ${ }^{9}$ and assist in restoring the ability to bathe independently or a carer to support bathing. Provision of bathing adaptions therefore may delay functional deterioration, improve health and quality of life, and postpone the need for other health and social care services. $^{3}$

We previously completed a feasibility RCT of major bathing adaptations (BATH-OUT) ${ }^{10}$ and a concurrent qualitative interview study. ${ }^{11}$ Sixty adults aged 65 and over, and their carers, were randomised to expedited provision of bathing adaptations versus routine waiting list control. Indicative findings showed improvements from baseline in both groups on all outcome measures following the installation of the accessible shower. Twenty-one of these older adults and five of their carers also took part in the qualitative interview study in which five themes were identified: ease of use, feeling safe, feeling clean; independence, choice and control; and confidence and quality of life. The findings were that the removal of physical barriers in the bathroom led to an improved sense of physical functioning which also impacted on the older adults' wider sense of confidence. The BATH-OUT qualitative findings ${ }^{11}$ were located within a framework of a moderated social model of disability, ${ }^{12}$ person-environment $\mathrm{fit}^{13}$ and environmental press theory ${ }^{14}$ postulating that people are disabled by barriers within their environment and that the environment needs to be optimally 'challenging' for the person in order to maintain optimum function.

The primary objective of this study was to revisit participants from the BATH-OUT qualitative interview study at 18-28 months on from the completion of their bathing adaptation in order to explore their lived experiences at this later time point. Ease of use had been highlighted as a key theme in the original BATH-OUT interview study, ${ }^{11}$ so we aimed to investigate whether usability and the other lived experiences reported in the original interview study were still evident in participants' narratives 2 years later. A secondary objective was to collect the outcome measures used in the feasibility RCT at this later time point. The rationale for collecting these measures was to support and add clarification to the themes of the qualitative interview data as the outcome measures used had previously been well connected to the themes identified in the original BATH-OUT interview study. ${ }^{11}$ Heywood and
Turner stated that multiple pieces of evidence must be combined in order to understand, ${ }^{15}$ for example, how an adaptation in the bathroom might prevent a fall in the living room. As such, collection of the outcome measure data served to encourage fuller understanding of the mechanisms at play, adding to the trustworthiness of the data and analysis. ${ }^{16}$

\section{METHOD}

\section{Study design and location}

This was an extended follow-up study consisting of a semi-structured qualitative interview supplemented with concurrent nested outcome assessments. The purpose was to explore the longer-term lived experiences of the older adults who had originally been interviewed following the installation of an accessible bathing facility. The nested method uses the quantitative data to support the primary qualitative data, adding depth to the older adults experiences, helping the reader to apply a broader context to the themes developed in the qualitative data. ${ }^{16}$

\section{Setting}

The BATH-OUT study was conducted within one local authority (City Council) housing adaptations service in England. Follow-up interviews took place at the site of the participant's choice, this was predominantly their home or the home of a relative.

\section{Population}

Potential participants for this extended follow-up study were limited to those who had previously taken part in the BATH-OUT feasibility RCT and concurrent qualitative interview study. Eligibility criteria were adults aged $\geq 65$ and referred to a local authority housing adaptations service for an accessible flush-floor shower. Reasons for referral for the accessible shower were varied across the original cohort. All participants received an assessment by an occupational therapy team member who determined that a level access shower was appropriate to meet the older adult's personal care needs. This would be due to their difficulties accessing the existing bathing facility or the unsuitability of bathing equipment or more minor adaptations to meet their needs.

\section{Recruitment}

All those who had been interviewed as part of the BATH-OUT qualitative interview study and had given consent to contact about further studies ( $\mathrm{n}=19$ older adults and $n=4$ carers) were approached. In the original BATH-OUT interview study participants were purposively sampled on gender, living arrangement and whether they were receiving assistance from an informal or formal carer. Participants who had lost the mental capacity to consent since the previous study were excluded. Participants were approached for interview irrespective of whether they were using their level access shower and/or were still living in the property in which it had been installed. 
Those who agreed to be contacted about the extended follow-up study were given an information sheet, study explanation and asked to sign a consent form.

\section{Data collection}

Semi-structured interviews were conducted with participants, and their carers, where appropriate. Interviews took place at a time and place of the participants choosing, which for all participants was their home residence. A pre-prepared topic guide was used which was drafted using the previous BATH-OUT topic guide with additional questions to encourage exploration of the longer-term experiences. A copy of the interview topic guide is included in the online supplemental appendix. Interviews were audio recorded using a digital recorder and transcribed verbatim. Older adults also completed the range of outcome measures collected in the BATH-OUT study. The outcome measures were: ShortForm 36 (physical and mental component scores), ${ }^{17}$ EuroQol EQ5D-5L, ${ }^{18}$ Adult Social Care Outcomes Toolkit (ASCOT),${ }^{19}$ Barthel Index (bathing question analysed as a separate outcome),${ }^{20} 0-100$ scale for perceived difficulty in bathing and the Short Falls-Efficacy Scale. ${ }^{21}$

\section{Data analysis}

\section{Qualitative}

Interviews were analysed using framework analysis in seven stages as outlined by Gale $e t a l .^{22}$ Recordings were transcribed verbatim by an external transcriber (stage 1) and then all transcripts were checked for accuracy by the authors. The authors then familiarised themselves with the entire dataset (stage 2) and coded three transcripts independently in duplicate. These transcripts were then compared to determine which sections had been highlighted for coding and the codes that had been applied to them (stage 3). These codes were than compiled and refined with strong consensus between the two reviewers creating an analytical framework (stage 4). This framework was then applied to the remainder of the transcripts by both reviewers (stage 5). Data extracted from the transcripts were then entered into the analytical framework using Microsoft Excel (stage 6). Stage 7 involved the interpretation of the data and the production of the report which was conducted jointly and agreed between the authors. The analysis was not informed by an a priori framework however the authors drew on the framework developed for the original BATH-OUT qualitative analysis. The framework for analysis of the extended follow-up interviews was developed iteratively, led by the data.

\section{Outcome measures}

Descriptive statistics were used to present the demographic characteristics of the participants of the study (older adult and carer). The results of outcome measures used at the extended follow-up time point and the results of the extended follow-up cohort in the original study at baseline, 3 months, 6 months and 9 months (means and SD) were also reported.

\section{Patient and public involvement}

The study was conceived with the assistance and approval of the BATH-OUT patient and public involvement (PPI) co-applicant and their input has informed and shaped the project and analysis. The PPI member was specifically involved with the selection of outcome measures used and advocated for face-to-face interviews and assessments rather than over the phone. A PPI group has now been formed and is made up of four members with personal experience of housing adaptations. They will guide and influence on-going BATH-OUT research work.

\section{FINDINGS}

Follow-up visits took place between 25 February 2019 and 15 March 2019; the mean time since completion of the adaptations to date of the follow-up visit was 23 months (SD 2.88). Audio recorded qualitative interviews took between $9 \mathrm{~min}$ and $32 \mathrm{~min}$ with an average length of $15 \mathrm{~min}$. Thirteen older adults and three carers were followed-up, though one carer chose not to complete the quantitative outcome measures. Eight of the 21 older adults interviewed in the original BATH-OUT study did not take part in the extended follow-up study. Two had not agreed to further contact and so were not approached. Two older adults who were approached declined a further follow-up, two had passed away, one had lost capacity and one was lost to follow-up due to having moved home.

Older adults ranged in age from 68 to 87 years old (mean: 76 , SD: 6.87). There were eight women and five men, with 10 living in council owned properties, a preponderance carried over from the original sample of interviewees. All of the older adults were retired and white British in ethnicity, 10 were living alone which again reflects the original sample. For the carer interviews two women took part, and one man with an age range of 50-75. The other two carers from the five which were included in the original BATH-OUT interview study were not followed up. One declined due to the death of their spouse and the other had not given consent for further contact. Of the carers who were included in the extended follow-up study, one lived with the older adult, one carer lived elsewhere with their children and the other was now living alone as the older adult for whom she cared had passed away since the previous interview. Demographic characteristics for the older adults and their carers are shown in table 1.

The majority of older adults reported that they were continuing to use their level access shower without any difficulties. However, three of the older adult's reported no longer using their level access shower. Two had moved to live with their sons since the previous interview where they only had an over bath shower. The third reported that she was not using the shower and had been choosing to strip wash (extracts are highlighted in the analysis). Another of the older adults reported initially having difficulty using the level access shower insofar as the shower head was set incorrectly and she did not know how to change it. This had since been resolved and she reported now using her shower without any difficulties. Finally, one 


\begin{tabular}{|c|c|c|}
\hline & $\begin{array}{l}\text { Older adult } \\
(n=13)(S D)\end{array}$ & $\begin{array}{l}\text { Carer }(\mathrm{n}=3) \\
(\mathrm{SD})\end{array}$ \\
\hline Age & $76(6.87)$ & $66.33(14.36)$ \\
\hline \multicolumn{3}{|l|}{ Gender } \\
\hline Male & 5 & 1 \\
\hline Female & 8 & 2 \\
\hline \multicolumn{3}{|l|}{ Marital status } \\
\hline Single & 2 & 1 \\
\hline Married & 1 & \\
\hline Widowed & 3 & 1 \\
\hline Divorced & 7 & \\
\hline Cohabiting & & 1 \\
\hline \multicolumn{3}{|l|}{ Property type } \\
\hline Council owned & 10 & 2 \\
\hline Owner occupied & 1 & 1 \\
\hline Housing association & 1 & \\
\hline Private rental & 1 & \\
\hline \multicolumn{3}{|l|}{ Living arrangement } \\
\hline Alone & 10 & 1 \\
\hline With other(s) & 3 & 1 \\
\hline With citizen (carers only) & & 1 \\
\hline \multicolumn{3}{|l|}{ Ethnicity } \\
\hline White British & 13 & 3 \\
\hline \multicolumn{3}{|l|}{ Employment } \\
\hline Retired & 13 & 2 \\
\hline Unemployed & & 1 \\
\hline
\end{tabular}

older adult who had participated in the original interview study had died, but his carer, an original study participant, chose to take part in this study as she was now using the shower herself.

Data were collected on whether the older adults were receiving assistance from a paid care worker or care from a family member or friend at the time of interview. At baseline of the original BATH-OUT study only one of the 13 older adults was receiving assistance from a paid care worker, this had increased to two older adults at the time of the extended follow-up. However, 12 of the 13 older adults reported having some form of assistance from friend or family carers at the baseline assessment, with four receiving assistance with personal care. This number had reduced to seven older adults receiving assistance from a friend or family carer at the time of the extended follow-up with only one receiving assistance with personal care.

\section{Qualitative: framework analysis}

Three themes were identified: the environment; autonomy with personal care; and wider occupation. Figure 1 illustrates how we have linked the themes conceptually. The

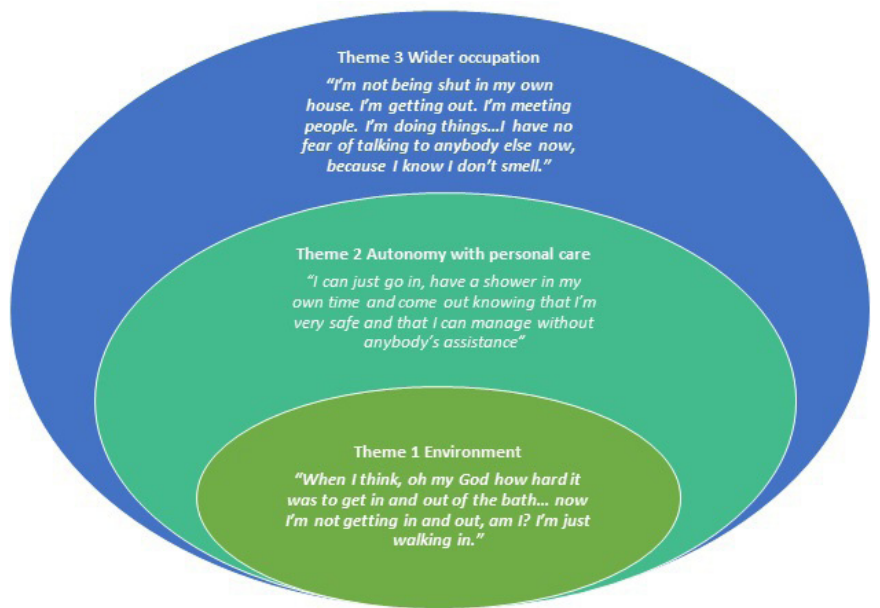

Figure 1 Overview of themes.

interface between the themes is shaped by the interaction between the environment (encompassing the physical and social environment), the person's autonomy with personal care (encompassing the older adult or the carers) and the impact of this on their wider occupation. This is consistent with environmental press theory ${ }^{14}$ and person-environment-fit ${ }^{13}$ and will be highlighted further in the Discussion section. Illustrative quotations from each of the themes are included in table 2.

\section{Theme 1: environment}

When I think, oh my God how hard it was to get in and out of the bath ... now I'm not getting in and out, am I? I'm just walking in. (Older Adult 11)

This theme relates to the environment and is split into two components: 'physical' and 'social'. The 'physical' subtheme predominantly encompasses the home environment, particularly the bathroom. The removal of the hazards from the previous bathroom were reported to have led to a wider, safer space enhancing the feeling of safety and reducing the fear of falling or injury. Fear was a component of the theme which generally related to reflections or recollections with many of the older adults describing how the installation of the shower had alleviated these fears in practical ways (quotation \#1). However, one older adult spoke about continued nervousness when using the new shower without a carer due to fear of falling. The older adults also looked to the future, again linked to the removal of the environmental barriers, reporting that there was an increased likelihood that they would be able to remain living in their own homes for longer because of the changes to the physical environment (quotation \#2).

The second subtheme 'social' is a smaller component of the environment theme and encompasses the impact on the carers, other members of the household and family members who did not live with the older adults. Some older adults and carers reported that other members of the household were using the adapted bathing facilities themselves which was reported to be beneficial to them (quotation \#3). Carers spoke particularly of how the 


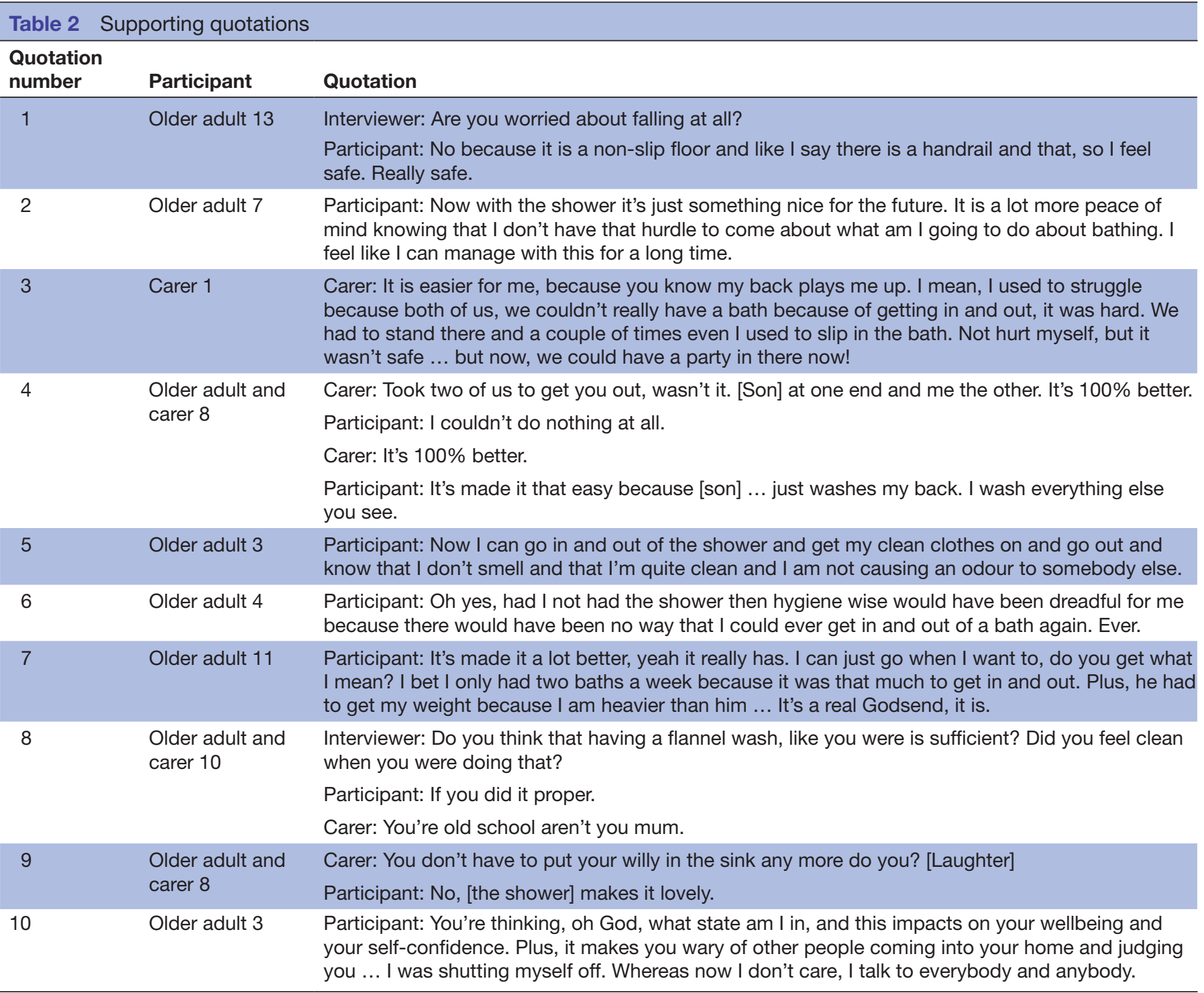

'usability' of the new bathroom had impacted on them by reducing the physical burden of assisting their relative to bathe, and also by making it easier for the participant to manage their personal care with less help (quotation \#4). Other older adults reported the installation of the adaptation had alleviated worry for their family members such that they were no longer as concerned about their safety at home. This was viewed positively by the older adults although some did highlight that their family contacted them less frequently now because they were less concerned about their safety.

\section{Theme 2: autonomy with personal care}

I can just go in, have a shower in my own time and come out knowing that I'm very safe and that I can manage without anybody's assistance (Older Adult 3)

This theme encompasses the direct impact of the bathing adaptation on the person when carrying out their personal care. It primarily comprises increased 'cleanliness' and greater 'choice and control'which combine to promote greater autonomy with personal care. 'Cleanliness' refers to the older adult having the ability to maintain a level of cleanliness which was adequate to them. Feeling clean was a theme in the original BATH-OUT analysis and it is apparent that it is the feeling associated with being clean which the older adults find of particular importance (quotation \#5). Choice and control relates to the older adults' autonomy to carry out their bathing routine at the time and frequency of their choosing. Several of the older adults reflected on how the adaptation had enabled them to continue to bathe as they would like (quotation \#6), though it did appear to be less related to bathing independently as found in the previous BATH-OUT analysis, ${ }^{11}$ and had become part of their routine and habit now that they had become accustomed to carrying out the activity on their own volition.

This theme was closely linked with having help from another person in the bathroom and the older adults' feelings about having that help (quotation \#7). This was also associated with enabling carers to manage and how the improved environment facilitated greater choice and 
control for them. In general, although the carers were willing to provide assistance, the older adults valued a greater degree of autonomy and less reliance on others which had been facilitated by the use of the level access shower. However, one older adult spoke about how she had not been using her shower because she said that carrying out a strip wash was satisfactory for her (quotation \#8), and she felt that this still afforded her adequate autonomy over her personal care routine.

\section{Theme 3: wider occupation}

I'm not being shut in my own house. I'm getting out. I'm meeting people. I'm doing things ... have no fear of talking to anybody else now, because I know I don't smell. (Older Adult 3)

This theme encompasses the wider effects of the bathing adaptation resulting from the changes in the physical and social environment which led to enhanced feelings of safety and the impact of increased autonomy in personal care. The wider occupation theme encompasses those aspects which appeared to occur as the indirect consequences of improvements in the physical and social environment cleanliness and choice and control. This enabled the older adults to carry out more of the things they needed to do such as toileting (due to the increased space and safety in the bathroom) or the things that they want to do, such as getting out and accessing their community.

It relates to the way the older adult interacts with the bathing adaptation and in particular how usable, and easy to use, they find it. Some of the older adults reflected on having to previously find alternative adaptive means of carrying out the activity of bathing which they no longer had to do (quotation \#9). This led to an increased sense of personal safety and 'mastery' that improved confidence affecting daily occupations inside and outside of the bathroom. One older adult highlighted that it particularly led to enhanced social interactions and reduced feelings of isolation (quotation \#10). Thus, it remains an important contributing factor to the older adults' level of confidence and mental well-being.

Older adults described specifically how the act of bathing was now associated with feelings of normalcy. It had become a routine activity which was part of their habituation and not something which required excessive planning or preparation. This contrasts with the 'chore' that it had been previously leading to feelings of elevated risk, trepidation and anxiety. Older adults spoke about now enjoying the act of bathing again, with some describing it as a leisure activity that had become a normal part of their daily lives.

\section{Links between the interview data and participant outcome measures}

All outcome measures have maintained or improved from baseline scores which were taken before the level access shower was installed.

The mean and SD for all outcome measures for older adults from the extended follow-up study, including those from the original study for comparison, are shown in table 3. The quantitative results act to support the primary qualitative findings and add clarification to the themes identified in the analysis.

The links between the individual domains of the ASCOT, a social care related quality of life measure and the qualitative analysis are particularly noteworthy. In addition to the overall improvement in ASCOT score shown in table 3, there were improvements in average scores across all the ASCOT domains as shown in table 4 . Each ASCOT domain can be scored 1-4 with lower scores indicating better outcomes. Analysis conducted by calculating the mean score of the individual domain responses shows that all older adults scored ' 1 ' for 'personal safety' at the extended follow-up, a change from 7 out of 13 at baseline. For 'personal cleanliness and comfort' (feeling clean), all participants scored ' 1 ' at the extended

Table 3 Outcome measures across all time points (older adult and carer)

\begin{tabular}{|c|c|c|c|c|c|}
\hline Older adult $(n=13)$ & $\begin{array}{l}\text { Baseline mean } \\
\text { (SD) }\end{array}$ & 3M mean (SD) & $6 \mathrm{M}$ mean (SD) & 9M mean (SD) & $\begin{array}{l}\text { Ext F-U mean } \\
\text { (SD) }\end{array}$ \\
\hline SF-36 physical component summary & $30.54(7.08)$ & $28.71(7.13)$ & $31.82(10.05)$ & $34.44(10.71)$ & $33.95(5.21)$ \\
\hline EQ-5D-5L & $0.48(0.22)$ & $0.49(0.22)$ & $0.62(0.25)$ & $0.67(0.21)$ & $0.74(0.15)$ \\
\hline EQ-5D perceived health & $55.38(16.77)$ & $51.92(22.41)$ & $62.62(24.42)$ & $69.23(23.79)$ & $69.23(19.98)$ \\
\hline Barthel Index & $17.62(2.60)$ & $17.69(2.75)$ & $17.69(2.87)$ & 17.69 (2.39) & $17.69(3.59)$ \\
\hline Perceived ease of bathing & $30.38(16.64)$ & 40.38 (36.2) & $86.15(23.02)$ & $97.31(5.99)$ & $90.77(18.47)$ \\
\hline Independent in bathing & 7 (53.9\%) & $8(61.5 \%)$ & $11(84.6 \%)$ & $11(84.6 \%)$ & $11(84.6 \%)$ \\
\hline Short Falls Efficacy Scale* & $18.38(4.59)$ & $17.15(4.78)$ & $15.38(4.75)$ & $14.62(4.91)$ & $11.15(3.74)$ \\
\hline
\end{tabular}

*Lower scores indicate better outcomes.

ASCOT, Adult Social Care Outcomes Toolkit. 
Table 4 Ascot score domain comparison

\begin{tabular}{llll}
\hline ASCOT domain* & Baseline mean (SD) & Extended Follow-Up mean (SD) & Change from baseline \\
\hline Control over daily life & $2(0.82)$ & $1.38(0.77)$ & -0.62 \\
Personal cleanliness and comfort & $1.84(0.69)$ & $1.15(0.55)$ & -0.69 \\
\hline Food and drink & $1.69(0.75)$ & $1.08(0.28)$ & -0.62 \\
Personal safety & $1.54(0.66)$ & $1(0)$ & -0.54 \\
Social participation and involvement & $1.93(0.73)$ & $1.36(0.5)$ & -0.57 \\
Occupation & $2.31(0.75)$ & $1.38(0.65)$ & -0.92 \\
Accommodation cleanliness and comfort & $1.46(0.52)$ & $1.08(0.28)$ & -0.38 \\
Dignity & $1.92(0.64)$ & $1.38(0.65)$ & -0.54
\end{tabular}

Each ASCOT domain can be scored 1-4 with lower scores indicating better outcomes. We carried out this analysis by calculating the mean score in each domain.

*Lower scores indicate better outcomes in the individual domains.

follow-up (except one who had moved to live with her son and was no longer using her accessible shower). All older adults reported either an increase in their feeling of 'control over daily life' or remaining at the same level as reported at baseline. Particular movement was also noted in the 'occupation' domain, a measure of how well the older adult is occupied in meaningful activities such as leisure activities, caring for others, or paid or unpaid work. This is consistent with the qualitative analysis which highlights the wider impact of the accessible shower on other aspects of self-care and leisure activities outside of the home. Overall, the domains driving the improvements appeared to be different for each of the participants.

\section{DISCUSSION}

This study suggests that the positive lived experience narratives reported immediately after the installation of the bathing adaptations were still evident at 23 months for older adults and their carers. The data also suggested that the impact on the older adult was now far more widespread than the direct consequences of being able to manage personal hygiene independently. This had a broader impact on their wider occupation and chiefly with activities outside of the home. Of the 13 older adults who took part in this extended follow-up study, three were no longer using the level access shower installed. The older adult who was choosing to strip wash spoke of how a lack of confidence in using the installed shower contributed to her preference. For the other two older adults a change in care needs led to their change in home circumstances. It is worth recognising that these two factors can be common in this age group and so could influence future research in this area.

The main strength of the study is that, to our knowledge, it is the first to explore the longer-term lived experiences of older adults who have had an accessible showering facility installed in their homes. It is possible that our previous qualitative interview study was influenced by the older adults' and carers' initial excitement or anxiety in the immediate time period after the level access shower had been installed; this study enhances the findings by conducting interviews again at a later time point once the initial novelty had worn off. Furthermore, the addition of the nested outcome assessments adds to the trustworthiness of the data and our analysis. The main limitation of this study is that it was conducted with a small sample of participants in one local authority area, the majority of whom lived alone, were relatively high functioning at baseline, and lived in publicly owned housing stock. Those who took part in this study volunteered to take part in the original qualitative interview study and agreed to be contacted about possible follow-up studies, thus they were particularly motivated to take part and to share their stories. It is possible that they are a particular group of bathing adaptations recipients for whom the adaptation was a particular 'success'. A further limitation is that the team who conducted the research undertook the original BATH-OUT study and inevitably were influenced by that research. However, transcripts were coded in duplicate and crosschecked at all stages of the process and the team engaged in a continual process of reflexive analysis.

On the whole, our findings are consistent with the wider literature in this field that housing adaptations are valued by recipients and have a positive impact on a range of outcomes such as accessibility and usability, ${ }^{23}$ increased feelings of safety, ${ }^{24}$ reduced risk of falls ${ }^{25}$ and improved physical and mental health. ${ }^{9}$ Furthermore, although the evidence suggests that the onset of disability in bathing is a significant warning point in the life course for older adults, ${ }^{6}$ there is a dearth of evaluative or interventional studies of interventions targeted at bathing. ${ }^{26}$ This study is consistent with the previous BATH-OUT qualitative findings ${ }^{11}$ that the domains underpinning social care related quality of life, particularly 'personal cleanliness', 'choice and control' and 'personal safety' are specifically pertinent for older adults who have had a major housing adaptation to their bathing facilities. This study has added the domain of 'occupation' as a particularly relevant medium term outcome. The comparison of the qualitative findings with the movement in the 'occupation' domain of the ASCOT demonstrates the potential for impact of adaptations within the bathroom on wider occupation, participation and feelings of safety. 
Overall there was a pervasive sense of 'normalcy' which penetrated across all the themes in the qualitative analysis; this was also evident in the reflexive account completed by the research assistant who conducted the interviews. With the installation of the accessible shower, older adults now considered the act of bathing as a normal activity which did not initiate feelings of anxiety, or concerns about safety, as it had done previously. The analysis from our previous qualitative interview study ${ }^{11}$ located the findings in relation to the theories of environmental press ${ }^{14}$ and person-environment-fit. ${ }^{13}$ This postulates that the fit between a person's competencies and the demands from their environment affect how well the individual is able to function; when problems are encountered modifications to the environment reduce the level of 'press' or maximise the level of 'fit' between the person and the environment to improve performance. In this study, the reduced challenges and risks within the bathroom appear to have been succeeded by feelings of increased autonomy in personal care and improved confidence and mastery in wider occupations.

This extended follow-up study has provided an indication of the medium-term impact of bathing adaptations for older adults and their carers. However, this study is small-scale and exploratory and further longitudinal research is required, as advocated by Powell et al, ${ }^{4}$ involving larger samples with quantitative outcomes and more diverse groups of participants. We suggest the measures used in this study would be suitable for use in further longitudinal studies. Notwithstanding the small scale, confirmation of these findings would have important implications for policymakers; bathing may often be considered 'low' priority by statutory services which may be erroneous considering the indicative wider impacts. Thus, further definitive evaluative studies are required on the effectiveness and cost effectiveness of bathing adaptations in the short, medium and long-term. There are also further unanswered questions around the adaptations process for privately owned properties as the majority of those included within this extended follow-up lived in publicly owned properties. We suggest that, due to the parallels seen between the qualitative and quantitative aspects of this study, future research should also examine the use of the ASCOT when evaluating the impact of other housing adaptions such as ramps, stair lifts or minor adaptations such as grab rails.

\section{CONCLUSION}

This research has indicated that the positive lived experiences reported immediately after the installation of the accessible bathing facility are still evident at up to 28 months later in this cohort of older adults. Our research highlights the importance of the relationship between the person and their physical environment, and how the removal of physical barriers can enhance the interaction between the two leading to improved functioning, feelings of safety, confidence and wellbeing. The continued ability of the older adults to manage their personal hygiene safely and with independence appears to affect their ability to interact with the wider social environment. However, the mechanisms appear to be different for each of the participants; the individual domain scores in the ASCOT findings show how for some older adults it was cleanliness which held the most importance whereas for others it was their safety. Overall, this research adds further support to using person-environment models in conducting further research on housing adaptations and we suggest that larger scale longitudinal studies across a more diverse population group would be of greatest benefit.

Acknowledgements We would like to thank the BATH-OUT investigators and project advisory group: Sue Adams OBE (chair), Stuart Belshaw, Tony Dawson, Professor Marilyn James, Professor Pip Logan, Julie Peart, Doug Stem, Professor Marion Walker MBE, and Claire Ward. We would also like to thank Paul Wetton, Gary Harvey and, Frank Nicholls from Nottingham City Council and the Nottingham City Council Consultation Group on Adaptations and OT services. We would also like to thank Dr Rachel Russel for suggesting that we should conduct this follow-up study. We would especially like to thank the older adults and their carers who participated in the study.

Contributors MG-D and PW were joint principal investigators for the study and they have jointly drafted the manuscript. MG-D led on the data acquisition and analysis. PW lead on the interpretation of the data. Both authors commented critically, read and approved the final manuscript.

Funding This work was supported by the Centre for Ageing Better (project code: BATH-OUT 1 Extended Follow-up Study). The views expressed in this publication are those of the authors and not necessarily those of the Centre for Ageing Better.

Competing interests None declared.

Patient consent for publication Not required.

Ethics approval Ethical approval was provided by Camberwell St Giles Research Ethics Committee (Ref: 19/L0/0069). Informed written consent was obtained from participants prior to study enrolment.

Provenance and peer review Not commissioned; externally peer reviewed.

Data availability statement № data are available. No additional study data is available.

Supplemental material This content has been supplied by the author(s). It has not been vetted by BMJ Publishing Group Limited (BMJ) and may not have been peer-reviewed. Any opinions or recommendations discussed are solely those of the author(s) and are not endorsed by BMJ. BMJ disclaims all liability and responsibility arising from any reliance placed on the content. Where the content includes any translated material, BMJ does not warrant the accuracy and reliability of the translations (including but not limited to local regulations, clinical guidelines, terminology, drug names and drug dosages), and is not responsible for any error and/or omissions arising from translation and adaptation or otherwise.

Open access This is an open access article distributed in accordance with the Creative Commons Attribution Non Commercial (CC BY-NC 4.0) license, which permits others to distribute, remix, adapt, build upon this work non-commercially, and license their derivative works on different terms, provided the original work is properly cited, appropriate credit is given, any changes made indicated, and the use is non-commercial. See: http://creativecommons.org/licenses/by-nc/4.0/.

ORCID iD

Miriam Golding-Day http://orcid.org/0000-0002-0700-5395

\section{REFERENCES}

1 Iwarsson S, Wahl H-W, Nygren C, et al. Importance of the home environment for healthy aging: conceptual and methodological background of the European ENABLE-AGE project. Gerontologist 2007;47:78-84

2 Allen K, Glasby J. 'The billion dollar question': embedding prevention in older people's services-ten 'high-impact' changes. Br J Soc Work 2013;43:904-24.

3 Royal College of Occupational Therapists. Adaptations without delay: a guide to planning and delivering home adaptations differently. London: Royal College of Occupational Therapists, 2019.

4 Powell J, Mackintosh S, Bird E, et al. The role of home adaptations in improving later life. London: Centre for Ageing Better, 2017. 
5 Keall MD, Pierse N, Howden-Chapman P, et al. Home modifications to reduce injuries from falls in the home injury prevention intervention (HIPI) study: a cluster-randomised controlled trial. Lancet 2015;385:231-8.

6 Gill TM, Guo Z, Allore HG. The epidemiology of bathing disability in older persons. J Am Geriatr Soc 2006;54:1524-30.

7 Gill TM, Allore HG, Han L. Bathing disability and the risk of longterm admission to a nursing home. J Gerontol A Biol Sci Med Sci 2006;61:821-5.

8 Heywood F. The health outcomes of housing adaptations. Disabil Soc 2004;19:129-43.

9 Heywood F. Money well spent: the effectiveness and value of housing adaptations. Policy Press, Joseph Rowntree Foundation, 2001.

10 Whitehead PJ, Golding-Day MR, Belshaw S, et al. Bathing adaptations in the homes of older adults (BATH-OUT): results of a feasibility randomised controlled trial (RCT) 11 medical and health sciences 1117 public health and health services. BMC Public Health 2018;18.

11 Whitehead PJ, Golding-Day MR. The lived experience of bathing adaptations in the homes of older adults and their carers (BATHOUT): a qualitative interview study. Health Soc Care Community 2019;27:1534-43.

12 Oliver M. The social model of disability: thirty years on. Disabil Soc 2013;28:1024-6.

13 Law M, Cooper B, Strong S, et al. The person-environmentoccupation model: a transactive approach to occupational performance. Canadian J Occup Ther 1996;63:9-23.

14 Lawton MP, Nahemow L. Ecology and the aging process. Washington, DC: American Psychological Association, 1973.

15 Heywood F, Turner L. Better outcomes, lower costs : implications for health and social care budgets of investments in housing adaptations, improvements and equipment : a review of the evidence.
University of Bristol, Office for Disability Issues, Department of Work and Pensions, 2007.

16 Creswell JW, Plano Clark VLP. Designing and conducting mixed methods research. 3rd edn. London: Sage Publications Ltd, 2017.

17 Ware JE, Sherbourne CD. The mos 36-item short-form health survey (SF-36). I. conceptual framework and item selection. Med Care 1992;30:473-83.

18 EuroQol Group. EuroQol - a new facility for the measurement of health-related quality of life. Health Policy 1990;16:199-208.

19 Netten A, Burge P, Malley J, et al. Outcomes of social care for adults: developing a preference-weighted measure. Health Technol Assess 2012;16:1-165

20 Collin C, Wade DT, Davies S, et al. The barthel ADL index: a reliability study. Int Disabil Stud 1988;10:61-3.

21 Yardley L, Beyer N, Hauer K, et al. Development and initial validation of the falls efficacy Scale-International (FES-I). Age Ageing 2005;34:614-9.

22 Gale NK, Heath G, Cameron E, et al. Using the framework method for the analysis of qualitative data in multi-disciplinary health research. BMC Med Res Methodol 2013;13:117.

23 Fänge A, Iwarsson S. Changes in accessibility and usability in housing: an exploration of the housing adaptation process. Occup Ther Int 2005;12:44-59.

24 Petersson I, Kottorp A, Bergström J, et al. Longitudinal changes in everyday life after home modifications for people aging with disabilities. Scand J Occup Ther 2009;16:78-87.

25 Clemson L, Mackenzie L, Ballinger C, et al. Environmental interventions to prevent falls in community-dwelling older people: a meta-analysis of randomized trials. J Aging Health 2008;20:954-71.

26 Golding-Day M, Whitehead P, Radford K, et al. Interventions to reduce dependency in bathing in community dwelling older adults: a systematic review. Syst Rev 2017;6:198. 